\title{
Intelligent wachsen? Hoffnungen wider die Tretmühle der Akkumulation
}

\author{
KLAUS DÖRRE
}

Die derzeit dominanten Formen des Wirtschaftens, Produzierens und Konsumierens bewirken, dass planetarische Belastungsgrenzen überschritten werden. An vorindustriellen Normwerten und Kipppunkten gemessen, wie sie ein Naturwissenschaftlerteam um Johan Rockström vorschlägt, befinden wir uns beim Klimawandel, der Artenvielfalt und dem Stickstoffkreislauf bereits jenseits der roten Linie. Übersäuerung der Ozeane, Ozonmangel, Frischwasserverbrauch, Landnutzung und atmosphärische Aerosolaufladung bewegen sich mit hoher Geschwindigkeit auf die Belastungsgrenzen zu. Insgesamt signalisieren diese Trends einen ökologischen Bruch im arbeitsvermittelten Stoffwechsel von Mensch und menschengemachter Natur. Hauptverursacher sind die Wachstumskapitalismen des globalen Nordens. Ein Viertel der Weltbevölkerung, vorwiegend des Nordens, konsumiert gegenwärtig drei Viertel der Ressourcen und erzeugt drei Viertel des Abfalls und der Emissionen. Um dem Süden eine Entwicklungschance zu erhalten, muss das Umsteuern in den kapitalistischen Zentren beginnen. Doch wie soll umgesteuert werden?

„Intelligent wachsen“ lautet das Credo neoschumpeterianischer Ansätze. Sie betrachten die ökologische Krise als Zukunftsmarkt, mit dessen Erschließung sich ein ergrünter Kapitalismus am eigenen Schopf aus dem Sumpf ziehen soll. Investitionen zur Erhöhung von Ressourceneffizienz und -effektivität gelten als Königsweg, um ökologische Nachhaltigkeitsziele im Wirtschaftssystem zu verankern. Doch sind das realistische Vorschläge? Gewiss: Ohne Innovationen ist z. B. das Umsteuern auf regenerative Energien nicht zu machen. Insofern sind grüne Technologien und Produkte eine notwendige, aber keine hinreichende Bedingung für ökologisches Wirtschaften. Die Technikfokussierung speist sich aus der Hoffnung, eine grüne Revolution könne erfolgreich sein, ohne die Kernstruktur des Wachstumskapitalismus qualitativ zu verändern. Gegen diese Sicht der Dinge sprechen wichtige Argumente.

\section{Geringe Amortisation "grüner“ Investitionen}

Erstens lässt sie die von finanzkapitalistischen Interessen geleitete Ausrichtung des Innovationsmodus außer Acht. Innoviert wird nur, sofern für Unternehmen Aussicht auf Markterweiterung, auf zusätzlichen und möglichst kurzfristigen Gewinn besteht. Das schuldengetriebene Wachstum der zurückliegenden Jahrzehnte hat eher Finanzinnovationen befördert als eine umfassende Ökologisierung der Produktionsökonomie eingeleitet. Offenbar sind Investitionen in grüne Technologien und Produkte riskant; häufig amortisieren sie sich, wenn überhaupt, nur langfristig. Die Gewinnaussichten sind, wie im Falle regenerativer Energien, begrenzt oder sie benötigen, wie ganzheitliche Mobilitätskonzepte, ein Finanzvolumen, das die Möglichkeiten selbst großer Unternehmen deutlich überschreitet. Aus diesem Grund tendiert auch die deutsche Exportwirtschaft dazu, ökologische Sprunginnovationen hinauszuzögern. Eher wird das Energieeinspeisegesetz zugunsten von Konzerninteressen korrigiert und werden Abgasrichtlinien der Europäischen Union den Wirtschaftlichkeitskalkülen der heimischen Autoindustrie angepasst, als dass ökologische Nachhaltigkeitskriterien die Richtung von Innovationen bestimmen könnten. Dazu passt, dass die aktuelle Prosperität der deutschen Exportwirtschaft wesentlich auf der Nachfrage vor allem chinesischer Mittelschichten beruht. Diese Milieus werden an hegemoniale Lebensstile herangeführt, die das Gegenteil ökologischer Nachhaltigkeit beinhalten.

\section{Fehlende Innovationsanreize}

Zweitens stoßen alle Versuche, das für kapitalistische Produktionsweisen charakteristische Kapitalüberschuss-Absorptionsproblem durch Markterweiterung zu lösen, derzeit an die Grenze limitierter zahlungsfähiger Nachfrage. Die Schwächung von Gewerkschaften, der ständige Druck auf Löhne und soziale Rechte, aber auch die Prekarisierung von Arbeits- und Lebensbedingungen sorgen dafür, dass organisierte Arbeitsinteressen nicht mehr als Produktivitätspeitsche und Innovationsanreiz wirken können. Teure ökologische Nischenprodukte eignen sich unter diesen Bedingungen bevorzugt für distinktiven Konsum; sobald Bioprodukte aus der Massenproduktion hervorgehen, steht ihre Nachhaltigkeit infrage. Hinzu kommt, dass die zunehmende soziale Polarisierung die Aufstiegsmobilität begrenzt und so potenziellen Unternehmern ein wichtiges Motiv nimmt, sich auf das Wagnis der Innovationskonkurrenz einzulassen. Stattdessen fördert die zunehmende Einkommens- und Vermögensungleichheit die Bereitschaft privilegierter Gruppen, ihr Geld in hochriskante Finanzgeschäfte zu investieren. Generell gilt, dass sich ökologische Nachhaltigkeitsziele in Gesellschaften mit ausgeprägten sozialen Ungleichheiten schwer durchsetzen lassen. Ungleichheit fördert positionalen Konsum, der auch von der Angst angetrieben wird, den Anschluss an gesellschaftliche Standards zu verpassen. Wir können unseren Lebensstil nicht einfach frei wählen, deshalb muss substanzielle Gleichheit, die positionalem Konsum seine Attraktivität nimmt, zu einem zentralen Ziel jeder Nachhaltigkeitsstrategie werden.

Die Chancen einer ökologischen Wende ließen sich drittens verbessern, wenn Staat und Politik zugunsten von Nachhaltigkeitszielen intervenieren würden. Dass Staaten dies können, hat sich während der globalen Krise 2008/09 gezeigt. Doch selbst dort, wo keynesianische Konjunkturprogramme wirksam wurden, fällt deren Ökobilanz desillusionierend aus. Trotz eines breiten Konsenses über die Notwendigkeit grüner Investitionen ist faktisch wenig passiert. Nur ein Bruchteil der staatlichen Mittel wurde für eine grüne Wirtschaft eingesetzt, und das Gros dieser Innovationen entfiel auf wenige Länder (Deutschland zählt dazu, wenn die Abwrackprämie als ökologische Investition gewertet wird). Ohne Druck aus der Zivilgesellschaft, 
ohne die Interventionen sozialer Bewegungen und ohne ökologisch inspirierte Wachstumskritik, so lässt sich schlussfolgern, sind nicht einmal neo-schumpeterianische Versionen eines ökologischen Umsteuerns realistisch.

\section{Reboundeffekte}

Würden sie dennoch verwirklicht, so sähen wir uns viertens mit einem grundlegenden Problem grüner Investitionen konfrontiert. Alles, was mit der Steigerung von Ressourceneffizienz und -effektivität zu erreichen ist, wird durch Reboundeffekte wieder zunichte gemacht. Kapitalistische Unternehmen funktionieren so wie Walmart: Tatsächlich hatte der Konzern bei der effizienten Ressourcennutzung Fortschritte gemacht. Doch das eingesparte Geld wurde für eine Expansion eingesetzt, deren destruktive Wirkungen die ökologischen Verbesserungen um ein Mehrfaches kompensierten. Geld, das eine mit ökologischen Motiven betriebene Rationalisierung erwirtschaftet, muss in der kapitalistischen Wirtschaft so rasch wie möglich reinvestiert werden, um neuen, zusätzlichen Gewinn zu produzieren. Das bedeutet erhöhten Stoffumsatz, steigenden Energiebedarf und erhöhte Belastungen für die Ökosysteme. Die Annahme, es genüge, die kapitalistische Effizienzlogik zugunsten von Nach- haltigkeitszielen einzusetzen, ist daher schlicht unrealistisch.

\section{Kapitalistischer Wachstumszwang vs. gesellschaftlich verantwortliche Produktion}

Damit kommen wir fünftens zum Kern des Problems. Ein auch nur annäherungsweise nachhaltiger Innovationsmodus wäre hochgradig auf Staatsintervention, zivilgesellschaftliche Bewegung, demokratische Kontrolle, öffentliches Eigentum, Aufwertung von Reproduktionstätigkeit, Ausgleich zwischen Nord und Süd, Egalitarismus und maßvollen Konsum angewiesen. Doch wäre eine Gesellschaft, die all dies leisten könnte, noch eine kapitalistische? Am Nachhaltigkeitsmaßstab gemessen, kommen wir wohl nicht an der Erkenntnis vorbei, dass sich ein Gesellschaftssystem mit eingeschriebenem Wachstumszwang nicht mit der Reproduktionsfähigkeit eines Planeten verträgt, dessen Ressourcen endlich sind.

Der Abschied von der Wachstumsillusion dürfte uns leichter fallen, wenn wir uns vergegenwärtigen, weshalb aus der schöpferischen Zerstörung, die Joseph Schumpeter analysierte, längst die kreative Konservierung unhaltbarer gesellschaftlicher Verhältnisse geworden ist. Der Wachstumskapitalismus ist zu einer Tretmühle mutiert, die sich nur noch bewegt, weil immer mehr Menschen immer mehr Energie aufwenden müssen, um nicht aus dem Rad herauszufallen. Wer es mit Nachhaltigkeitszielen ernst meint, muss diese Tretmühle grundsätzlich infrage stellen. Das geht nur, wenn die Gesellschaft jenseits privater Gewinninteressen und hoffentlich mit gewerkschaftlicher Hilfe auf das "Was“ und das „Wozu“ der Produktion Einfluss nimmt. Eine neue Wirtschaftsdemokratie wäre nötig, um Nachhaltigkeitsinnovationen zu ermöglichen, die diesen Namen verdienen. Wer die notwendige Grüne Revolution auf eine technokratische Vision reduziert, wird indessen nicht einmal das Tempo drosseln, mit dem sich die Tretmühle der Akkumulation unerbittlich in die falsche Richtung bewegt.

\section{AUTOR}

KLAUS DÖRRE, Dr. habil., ist Professor für ArbeitsIndustrie- und Wirtschaftssoziologie und Ko-Direktor der DFG-Kollegforschergruppe „Postwachstumsgesellschaften" an der Friedrich-Schiller-Universität Jena. Arbeitsschwerpunkte: Prekarität, Theorie kapitalistischer Landnahmen.

klaus.doerre@uni-jena.de 